\title{
Laser-induced force on a microfluidic drop: Origin and magnitude
}

\author{
Emilie Verneuil, ${ }^{*} \dagger, \S$ María Luisa Cordero, ${ }^{\dagger}$ François Gallaire, ${ }^{\ddagger}$ and Charles N. \\ Baroud $^{* \dagger} \dagger$
}

Laboratoire d'Hydrodynamique (LadHyX), Ecole Polytechnique, 91128 Palaiseau, France, and Laboratoire J.A. Dieudonné, Université de Nice Sophia-Antipolis, 06108 Nice, France

E-mail: emiliev@seas.upenn.edu; baroud@ladhyx.polytechnique.fr

\begin{abstract}
The localized heating produced by a tightly focused infrared laser leads to surface tension gradients at the interface of microfluidic drops covered with surfactants, resulting in a net force on the drop whose origin and magnitude are the focus of this paper. First, by colocalization of the surfactant micelles with a fluorescent dye, we demonstrate that the heating alters their spatial distribution, driving the interface out of equilibrium. This soluto-capillary effect opposes and overcomes the purely thermal dependence of the surface tension, leading to reversed interfacial flows. As the surface of the drop is set into motion, recirculation rolls are created outside and inside the drop that we measure using time-resolved micro-Particle Image Velocimetry. Second, the net force produced on the drop is measured using an original microfluidic design. For a drop $300 \mu \mathrm{m}$-long and $100 \mu \mathrm{m}$ wide we obtain a force of 180

\footnotetext{
${ }^{\dagger}$ Laboratoire d'Hydrodynamique (LadHyX), Ecole Polytechnique, 91128 Palaiseau, France

${ }^{\ddagger}$ Laboratoire J.A. Dieudonné, Université de Nice Sophia-Antipolis, 06108 Nice, France

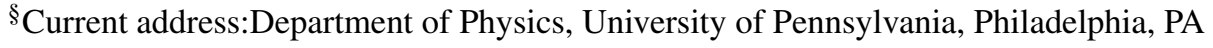


$\mathrm{nN}$ for a laser power of $100 \mathrm{~mW}$. This micro-dynanometer further shows that the magnitude of the heating, which is determined by the laser power and its absorption in the water, sets the magnitude of the net force on the drop. On the other hand, the dynamics of the force generation is limited by the time scale for heating which has independently been measured to be $\tau_{\Theta}=4 \mathrm{~ms}$. This time scale sets the maximum velocity that the drops can have and still be blocked, by requiring that the interface passes the laser spot in a time longer than $\tau_{\Theta}$. The maximum velocity is measured at $U^{\max }=0.7 \mathrm{~mm} / \mathrm{s}$ for our geometric conditions. Finally, a scaling model is derived that describes the blocking force in a confined geometry as the result of the viscous stresses produced by the shear between the drop and the lateral walls.

\section{Introduction}

Surface tension gradients have recurrently been proposed as a way to apply a force on a drop or a bubble in order to manipulate it. The flows that are produced by these gradients (Marangoni flows) can be of thermal origin, in the case of thermocapillary flows, ${ }^{1-5}$ or of chemical origin through gradients of surfactant coverage of the interface, in the case of solutal Marangoni flows. ${ }^{6,7}$ In both cases, the unequilibrated surface tension applies a stress along the interface which leads to the motion of the fluid. Recently these approaches have acquired increasing immediacy with the emergence of droplet-based microfluidics, for which there have been many proposals for fluid actuation based on the manipulation of surface stresses. ${ }^{8}$

The generation of a net force by thermocapillary flow was first demonstrated by Young et al. on air bubbles located between the anvils of a machinist's micrometer. ${ }^{1}$ The anvils were held at different temperatures, leading to a thermocapillary flow along the surface of the bubble which also induced a flow from the hot to the cold regions in the external fluid. In reaction to this external flow, the drop "swims" up the temperature gradient towards the hot side. The magnitude of the forces generated in those experiments can be calculated by balancing the buoyancy force acting on a bubble of diameter $200 \mu \mathrm{m}$, i.e. $40 \mathrm{nN}$, for a temperature gradient of $80^{\circ} \mathrm{C} / \mathrm{cm}$ or 
equivalently $1.6^{\circ} \mathrm{C}$ across the bubble.

Recent studies have revisited bubble migration in a temperature gradient in a confined geometry, ${ }^{4,9}$ where a similar mechanism drives the bubble towards the hot region at a velocity that increases linearly with the temperature gradient. However, obtaining a quantitative value of the force acting on the bubble in this case is difficult due to the effects of confinement which couple the bubble shape, the flow, and the net force. In practical situations, further complications appear when the thermocapillary effect is conjugated with the transport of surfactant molecules. ${ }^{10,11}$ Indeed, the interface motion advects the surfactant molecules and can lead to the accumulation of these molecules at the stagnation points on the drop, leading to additional gradients of stress. These combined phenomena were observed to slow the migration of a drop in a thermal gradient ${ }^{10}$ or to inhibit the onset of thermocapillary convection along a flat interface. ${ }^{11}$

A recent series of articles has explored the use of laser heating to manipulate drops of water in oil travelling inside microfluidic channels. ${ }^{12-14}$ The laser in these experiments is chosen to heat only the water phase, it remains stationary and is focused to a waist of a few microns, small compared to the typical drop size $(100 \mu \mathrm{m})$. When the leading edge of the drop reaches the position of a laser, the water is heated by absorption of the light and a repulsive force pushes the drop away from the hot spot, i.e. in the direction opposite to the pure thermocapillary case discussed above. ${ }^{1,4}$ This was explained in a previous work ${ }^{12}$ where observation of the fluid motion showed that the flow was directed towards the hot region along the interface, indicating an increase of the surface tension at the hot spot location.

These observations motivate the present study on the physical and physico-chemical mechanisms responsible for the forcing of microdrops subjected to local heating. Below, we address the two questions of the origin and magnitude of this repulsive force, relying on experimental measurements in a specifically designed microfluidic geometry shown in Figure 1 in which water drops are formed and transported by an oil flow. A focused infrared laser is used to apply 
localized heating on the water-oil interface and images are recorded using a fast camera.

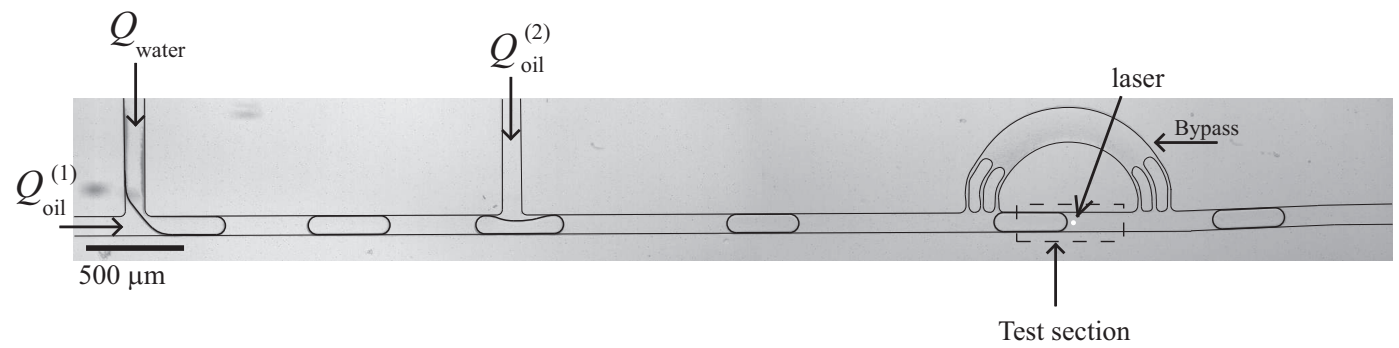

Figure 1: Microscope image of the microfluidic device. Drops of water are emitted in oil at the left T-junction. The total flow rate is adjusted downstream by a second oil entry. The drop is trapped by the infra-red laser in the test section while oil can flow into the bypass.

\section{Role of the surfactant}

The crucial role of the surfactant is revealed by a series of experiments performed with pure water drops in pure hexadecane: Without surfactant, the laser is never seen to hold the water/oil interface even at the highest laser power. Furthermore, tracking some droplets that are atomized by the heating shows that the laser induces rapid flows at the interface, directed from the hot parts to the cold parts. These observations agree with the classical thermocapillary effect but contrast with the observations made in the presence of surfactant that are reported by Baroud et al. ${ }^{12}$ and in the present study, where the flows are in the opposite direction. In the following, we explore the effects associated with the addition of surfactants to the water-oil system.

\section{Surface tension at equilibrium}

The evolution of the interfacial tension between a hexadecane/Span80 solution and pure water is first explored using the pendant drop technique. A pendant drop of $100 \mu \mathrm{L}$ ultra pure water is emitted at the tip of a vertical cylindrical pipette which is immersed in a thermostated oil bath. 


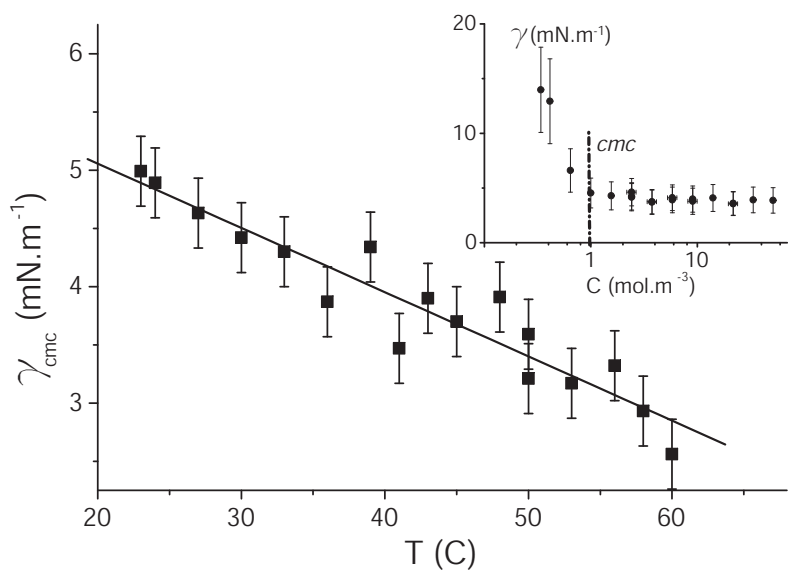

Figure 2: Surface tension of a water in hexadecane drop above the $\mathrm{cmc}(\mathrm{C}=36 \mathrm{cmc})$ as a function of the bulk oil temperature, fitted with a linear law (solid line). Inset: Surface tension as a function of the concentration $C$ of surfactant at $T=28{ }^{\circ} \mathrm{C}$. The $\mathrm{cmc}$ is estimated at $1 \mathrm{~mol} \cdot \mathrm{m}^{-3}$.

After an equilibration delay of 3 minutes, ${ }^{15}$ the interfacial tension $\gamma$ is obtained from the drop shape,${ }^{16}$ taking into account the temperature dependence of the liquid densities. ${ }^{17}$

The temperature dependence of surface tension is measured by increasing the temperature of the oil bath from 23 to $60^{\circ} \mathrm{C}$, for a bulk surfactant concentration equivalent to the microfluidics experiments $C=36 \mathrm{~mol} . \mathrm{m}^{-3}$ ( $\left.2 \mathrm{wt} \%\right)$. The interfacial tension is found to linearly decrease with the temperature, as shown in Figure 2, with a slope $\gamma_{T}=-55 \pm 8 \mu \mathrm{N} / \mathrm{m} / \mathrm{K}$. This measurement rules out the hypothesis of an anomalous thermal dependence of the surface tension that could reverse the sign of the interface velocity compared to the classical thermocapillary effect.

In a second series of experiments, the surfactant concentration $C$ was varied from $52 \mathrm{~mol} . \mathrm{m}^{-3}$ to $0.3 \mathrm{~mol} . \mathrm{m}^{-3}$ at $T=28{ }^{\circ} \mathrm{C}$. The value of the variation of $\gamma(C)$ is shown in the inset of Figure 2, which also gives a measure of the critical micellar concentration $\left(\mathrm{cmc}=1 \mathrm{~mol} . \mathrm{m}^{-3}\right.$ or $0.054 \mathrm{wt} \%)$, the point where $\gamma$ becomes independent of the surfactant concentration. These measurements are consistent with published values. ${ }^{15}$

We use the Gibbs adsorption equation to describe the equilibrium behavior of Span 80 at the water/hexadecane interface for concentrations below the $\mathrm{cmc}$, 


$$
d \gamma=-R T \Gamma d \ln C
$$

and a Langmuir isotherm

$$
\Gamma(C)=\Gamma_{\infty} \frac{K_{L} C}{1+K_{L} C}
$$

which lead to

$$
\gamma(C)=\gamma_{0}-R T \Gamma_{\infty} \ln \left(1+K_{L} C\right)
$$

where $R$ is the ideal gas constant, $T$ is the absolute temperature, $K_{L}$ is the equilibrium adsorption constant, $\Gamma_{\infty}$ is the maximum surface concentration of the surfactant and $\gamma_{0}$ is the interfacial tension of the water/hexadecane surface without surfactant. Equation (3) is found to fit the surface tension data collected by Campanelli et al. ${ }^{15}$ on the same system, at $\mathrm{T}=18^{\circ} \mathrm{C}$ and for a range of concentrations below $\mathrm{cmc}$. The fit (not shown) provides the following set of parameters: $\Gamma_{\infty}=4.10^{-6} \mathrm{~mol} \mathrm{~m}{ }^{-2}, K_{L}=110 \mathrm{~mol}^{-1} \mathrm{~m}^{3}$ and $\gamma_{0}=52.5 \mathrm{mN} \mathrm{m}^{-1}$. This result has three major implications: First, the fact that a Langmuir isotherm applies indicates the existence of an adsorption energetic barrier at room temperature; only the molecules having a high enough energy can be adsorbed or desorbed. ${ }^{15}$ Second, since $K_{L} \times c m c \gg 1$, Eq. (2) yields an equilibrium surface concentration that is close to $\Gamma_{\infty}$ at the $c m c$ and above. Finally, the derivative of $\gamma$ with respect to $\Gamma$ is

$$
\frac{\partial \gamma}{\partial \Gamma}=\frac{-R T \Gamma_{\infty}}{\Gamma_{\infty}-\Gamma}
$$

which states that the surface tension is sensitive to small variations of $\Gamma$ when $\Gamma \simeq \Gamma_{\infty}$ and that $\gamma_{\Gamma}$ has a highly negative value.

\section{Spatial surfactant redistribution}

Since the variations of surface tension with temperature do not account for the observed increase in interfacial tension at the hot spot, we now explore the dynamics of surfactant transport as a 
possible mechanism. Indeed, surfactant molecules can be transported by the hydrodynamic flow, diffusion, or thermophoresis. The effect of these transport mechanisms is to produce unbalanced surface coverage of the water-oil interface with surfactant molecules, which can lead to solutal Marangoni stresses on the interface. ${ }^{18}$ Accordingly, we recall some fundamentals of the dynamic variation of surface tension. In a quiescent and isothermal fluid, the partition of surfactants between the bulk and an interface is commonly modeled by a two-step process: ${ }^{19}$ First, the molecules diffuse from the bulk to the subsurface, which is a depleted transition layer located near the interface. The size of the subsurface is given by the ratio between the bulk and the interfacial concentration: $\xi=\Gamma / C \approx \Gamma_{\infty} / C$, which we calculate to be $\xi=4 \mu \mathrm{m}$ in the conditions of our experiments. The second step is the transfer of the molecules from this subsurface to the interface. The dynamics of this transport depends on the amount of coverage of the interface, with energetic barriers to adsorption appearing for crowded interfaces. The standard picture is that micelles also contribute to providing molecules to the interface in the case of non-ionic surfactants such as Span80. ${ }^{19}$ They can leak monomers on the microsecond timescale and thus play a role in the dynamic variations of the surface coverage and the exchanges between the interface and the subsurface. ${ }^{20}$ The relevant concentration that determines the variations of the surface tension, when out of equilirium, is therefore the subsurface concentration $C_{s}$ taking into account both the monomers and the micelles. In the framework of the Langmuir isotherm of Eq. (2), the rate of adsorption is proportional to $C_{s}$ and to the number of available interfacial sites $\left(\Gamma-\Gamma_{\infty}\right)$. The desorption rate, on the other hand, is proportional to the surface coverage $\Gamma$ and doesn't depend on $C_{s} \cdot{ }^{18}$

The spatial variations of the micelle concentration in the oil phase were measured by marking them with Rhodamine 101, a fluorescent dye (200 mM obtained by dilution in HEPES buffer $1 \mathrm{mM}, \mathrm{pH}=7$, from a $10 \mathrm{~g} / \mathrm{L}$ stock-solution in DMSO). Although Rhodamine is insoluble in pure hexadecane, it was found to dissolve in the organic phase at high concentration of Span 80 (36fold $\mathrm{cmc}$ ). This is attributed to an aggregation of the Rhodamine molecules within the micelles, 

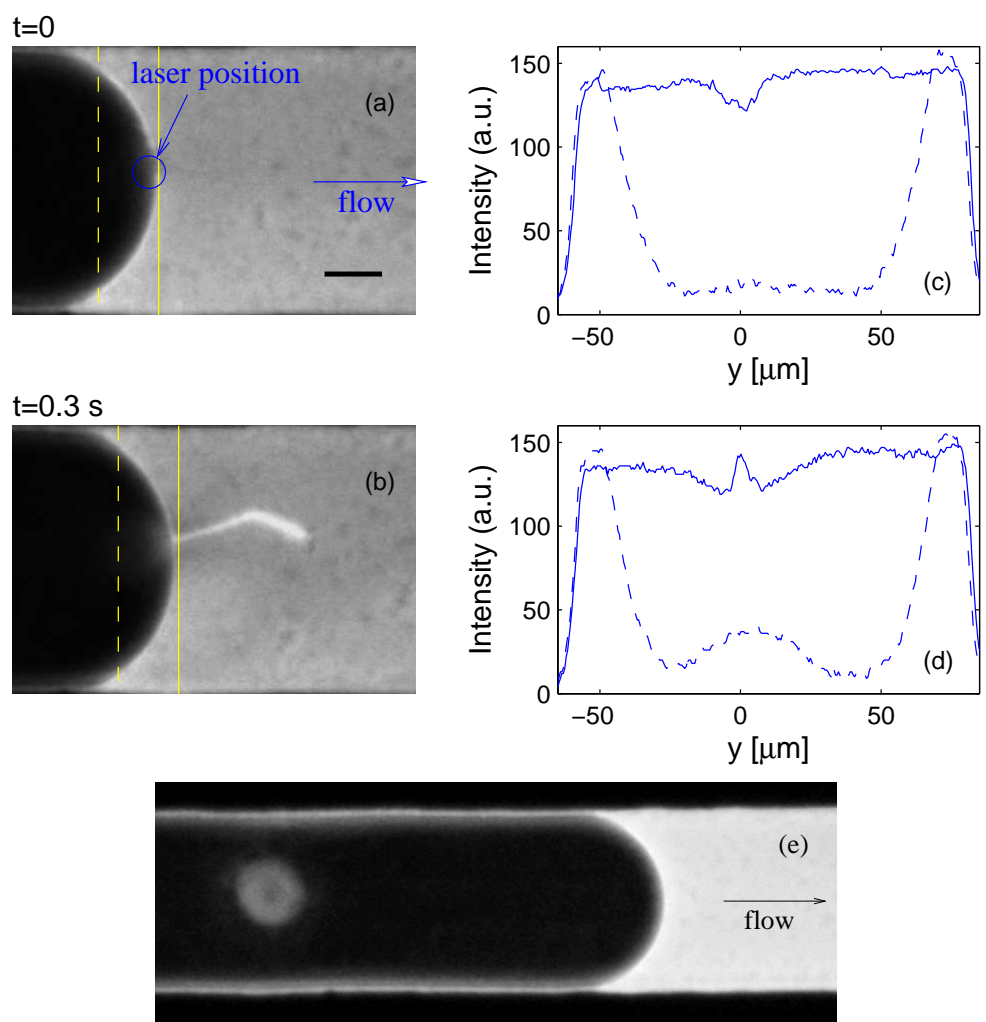

Figure 3: $(\mathrm{a}, \mathrm{b})$ Fluorescence images of a drop held by the laser in the test section at times $t=0$ (a) and $t=0.3 \mathrm{~s} \mathrm{(b)}$. The scale bar represents $30 \mu \mathrm{m}$. (c,d) Intensity profiles taken along the plain and dashed lines of images $(\mathrm{a}, \mathrm{b})$; (e) Image taken after the drop detachement showing the ring of adsorption of surfactant on the PDMS.

since Rhodamine can act as a co-surfactant, owing to its aromatic hydrophobic structure ending in a polar zwitterionic head. This allows the use of Rhodamine as a tracer for the micelles, especially that Rhodamine 101 fluorescence is insensitive to temperature variations. ${ }^{21,22}$

The behavior of the fluorescence field is shown in Figure 3a, which shows a drop beginning to interact with the laser. A dip in the fluorescence intensity is observed in the initial moments after the drop begins to be heated by the laser. This local decrease is more visible in Figure $3 \mathrm{c}$, where the fluorescence intensity is plotted along the solid line of Figure 3a. The spatial extent of the dip in fluorescence is about $10 \mu \mathrm{m}$, consistent with the size of the heated region at early times. The fluorescent intensity is also shown in Figure $3 \mathrm{~b}, \mathrm{~d}$ for $t=300 \mathrm{~ms}$, long after the drop has come to 
a complete halt. The decrease in fluorescence intensity remains but is now spread over a distance of about $\pm 20 \mu \mathrm{m}$, still consistent with the size of the hot region $(\sigma)$ at those times. However, a bright "jet" now crosses the depleted region and is advected by the mean flow away from the interface, indicating a major redistribution of the micelles in the flow. Moreoever, an increase in fluorescence is also observed upstream of the laser, corresponding to micelles being transported in the oil layer between the water drop and the PDMS wall, as shown by the intensity profiles measured along the dashed lines.

Finally, a fluorescent ring is observed at the location of the laser once the drop has detached and begun to move, as shown in Figure 3e. This ring is located on the surface of the PDMS and it can be washed away by flowing the oil for a few minutes. It should be noted that the jetting and the ring at the channel surface can be observed without the fluorescent marking, using diascopic illumination. Fluorescence images with Rhodamine allow a sharper contrast and a more quantitative measure of the phenomena.

Taken together, these observations show that the initial surfactant concentration is redistributed in a non-uniform way during the blocking of the drop by the laser, with the gradient occuring on a scale comparable with the scale of the temperature gradient at long times, $\sigma \simeq 20 \mu \mathrm{m}$. These length scales are comparable to the depth of the subsurface $\xi$ calculated above, implying that the gradients created by the laser are likely to modify the subsurface concentration in a way to affect the surface tension locally. The hydrodynamic effects of this surface tension variation are studied next.

\section{Hydrodynamics and force balance}

We now turn to evaluating the force that the laser heating is capable of producing on a microfluidic drop. A static force balance provides the magnitude of the laser-induced forcing while its dynamic evolution is discussed in light of the flow kinematics. 


\section{Steady state force}

If the drop is held stationary, a force balance must exist between the hydrodynamic effects that push it to the right and the force of thermocapillary origin that resists its motion. The design of the microchannel with a bypass section of resistance $R^{b}$ allows us to calculate the pressure difference between the left and right ends of the test section in the same way as for the voltage drop in an electrical circuit. Indeed, if the flux is all directed into the bypass when the drop stops, then the pressure difference linearly increases with the flux $P=R^{b} Q$. Since the same pressure difference applies on both the test section and the bypass, the total force that acts on the drop is

$$
F=R^{b} S Q
$$

where $S \simeq w \times h$ is the projected surface of the drop, nearly equal to the cross-section of the channel. Using the calibrated value of $R^{b}$, this relation predicts the magnitude of the force pushing a stationary $\operatorname{drop} F=180 \mathrm{nN}$ for $Q=5 \mathrm{~nL} / \mathrm{s}$.

This evaluation of the force must be corrected by the fact that some fluid manages to flow in the corners between the interface and the walls. ${ }^{23,24}$ This leakage can be taken into account by associating a resistance $R^{d}$ to the drop of length $L^{25,26}$ in series with the resistance $R^{t}=$ $1.710^{12} \mathrm{~Pa} \mathrm{~s} \mathrm{~m}^{-3}$ due to the shortened test channel of length $L_{t}-L$. Then, by analogy with an electrical circuit, the total flow rate $Q$ and the test channel flow rate $Q_{t}$ are related by balancing the pressure differences in the bypass and in the test channel: $Q / Q_{t}=\left(R^{b}+R^{t}+R^{d}\right) / R^{b}$. The final term that must be estimated is $R^{d}$, which is obtained from the measurements of the drop velocity in the test channel $\left(U_{0}\right)$ before interacting with the laser at various $Q$. By assuming that the drop travels at the mean flow velocity, namely $Q_{t}=U_{0} S$, the slope of the line $U_{0}(Q)$ yields $R^{d}=1210^{12} \mathrm{~Pa} \mathrm{~s} \mathrm{~m}^{-3}$. Finally, the pressure difference across the drop is derived as $R^{d} Q_{t}$ and the modified force $F^{\prime}$ can be estimated as this pressure difference times the channel cross-section: 


$$
F^{\prime}=\frac{R^{b} R^{d}}{R^{t}+R^{b}+R^{d}} S Q
$$

We calculate $F^{\prime}=100 \mathrm{nN}$ for a flow rate $Q=5 \mathrm{~nL} / \mathrm{s} . F^{\prime}$ can be taken as the lower limit for the force the laser has to exert to block the drop, while $F$, which can be recovered from Eq. (6) for an infinite value of the drop resistance, can be taken as an upper bound.

\section{(a)}

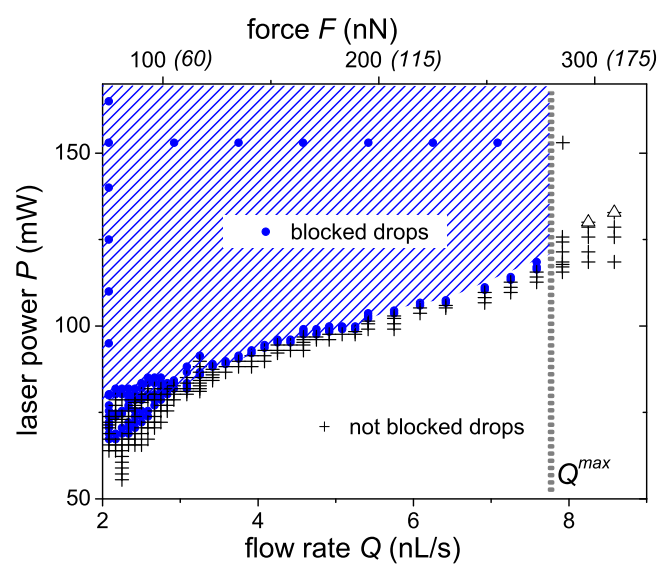

Figure 4: (a) Phase diagram showing the blocked drops for different laser powers as a function of the flow rate $Q$ : full circles indicate drop that are blocked and crosses the non-blocked ones. Triangles correspond to cases where water boils. The secondary $\mathrm{x}$-axis shows a conversion of $Q$ into the total force $F\left(F^{\prime}\right)$ applied by the laser, taken from Eqs. 5 and 6, respectively. (b) Minimum power required to block a drop, $P^{\mathrm{min}}$, as a function of the laser width $\omega$ normalized by the waist $\omega_{0}$. $\omega$ was varied either by increasing the sample to objective distance along the optical axis $(z>0)$ or by decreasing it $(z<0)$. The flow rate is $Q=3.75 \mathrm{~nL} / \mathrm{s}$.

Systematic measurements of the minimum laser power $P^{\min }$ required to stop a drop with a given flow rate were performed and the resulting diagram is shown in Figure 4a. An arbitrary criterion was chosen to distinguish the drops that were blocked from those that escaped by requiring a minimum blocking time of $200 \mathrm{~ms}$. As expected, $P^{\mathrm{min}}$ increases as the flow rate is increased and the corresponding values of the force, obtained from Eqs. (5) and (6), display values in the range of a few hundred $\mathrm{nN}$. Furthermore, a maximum flow rate $Q^{\text {max }}=7.9 \mathrm{~nL} / \mathrm{s}$ is observed above which drops cannot be held at any laser power. Finally, it is important to note 
that blocking longer drops requires a lower value of $P^{\min }$ than for shorter drops (not shown).

In a separate series of experiments, the variations of $P^{\min }$ with the laser spot size $\omega$ are explored by defocusing the laser, as described in the experimental section, while keeping the flow rate constant at $Q=3.75 \mathrm{~nL} / \mathrm{s}$. The effect of an increase in the laser width is known from our previous study to decrease the temperature at the hot spot while keeping the typical heating time constant. ${ }^{22}$ We observe here that the minimum power required to block a drop increases as the beam size is increased, as shown in Figure 4b.

\section{Transients before coming to a complete halt}

The steady state force computed above (Eq. 5) gives only a partial view of the thermocapillary blocking process. In the following, we turn to the transients that precede the blocking by analyzing the evolution of the fluid velocities measured by the PIV. Figure 5a displays a superposition of thirty successive images $(120 \mathrm{~ms})$ of the tracer particles, showing the presence of recirculation rolls inside the stationary drop. The corresponding velocity field is shown in Figure 5b, where the calculated field is averaged over 1035 images. The PIV gives an accurate measure of the velocities away from the hot region but the values near the laser position are poorly computed due to the very high accelerations near the hot spot. Nonetheless, we observe that the interface of the drop is set into a motion directed toward the laser position as the drop comes to a halt, and the structure of the rolls is clearly recovered. We find that the positions of the roll centers do not depend on time nor on the total flow rate. They are located at a distance $d=17 \pm 3 \mu \mathrm{m}$ from the drop interface and at a distance of $25 \mu \mathrm{m}$ from the channel lateral walls, suggesting that the roll position is set by the channel geometry.

The drop velocity was determined by spatially averaging the computed velocities over the whole field, as shown in Figure 6 for four values of the flow rate. As expected, the velocity prior to interaction with the laser $U_{0}$ increases with $Q$. Upon arriving at the laser position, drops slow to a halt over a few ms and remain stationary for long times. The drop corresponding to 


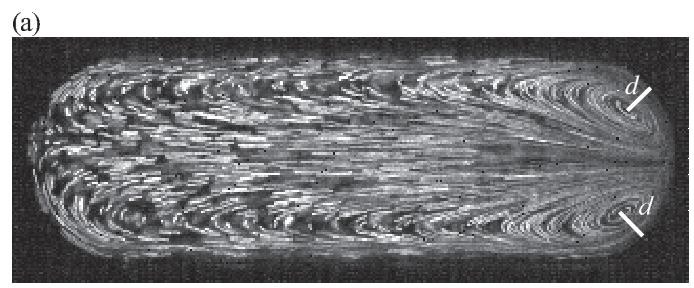

$100 \mu \mathrm{m}$

(b)

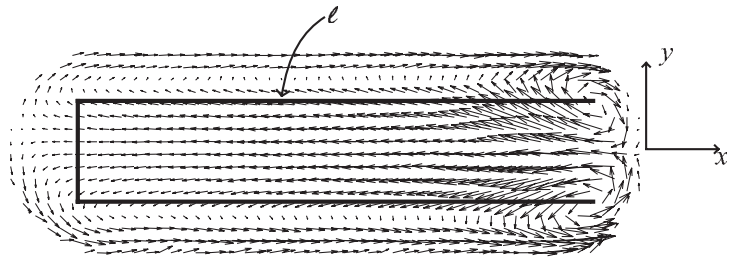

(c)

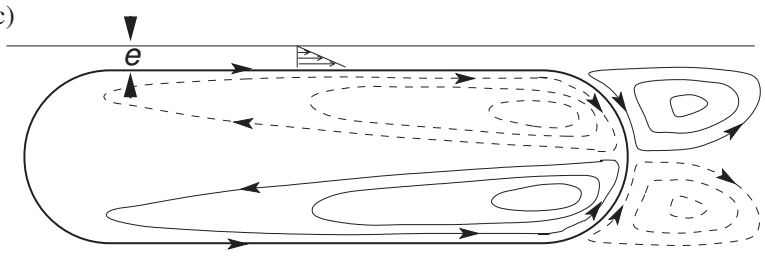

Figure 5: (a) Superposition of fluorescence images of a drop held by the laser, showing a pair of stationary rolls. (b) Time-averaged velocity field inside the drop in the steady state. The line $\ell$ is used for the calculation of the flux which must return through the line $d$. (c) Schematic of the flow and leakage flow in the thin films between walls and interface, drawn artificially larger. 
$Q^{\max }=7.9 \mathrm{~nL} / \mathrm{s}$, however, escapes after it has been stopped for $80 \mathrm{~ms}$ at the laser position.

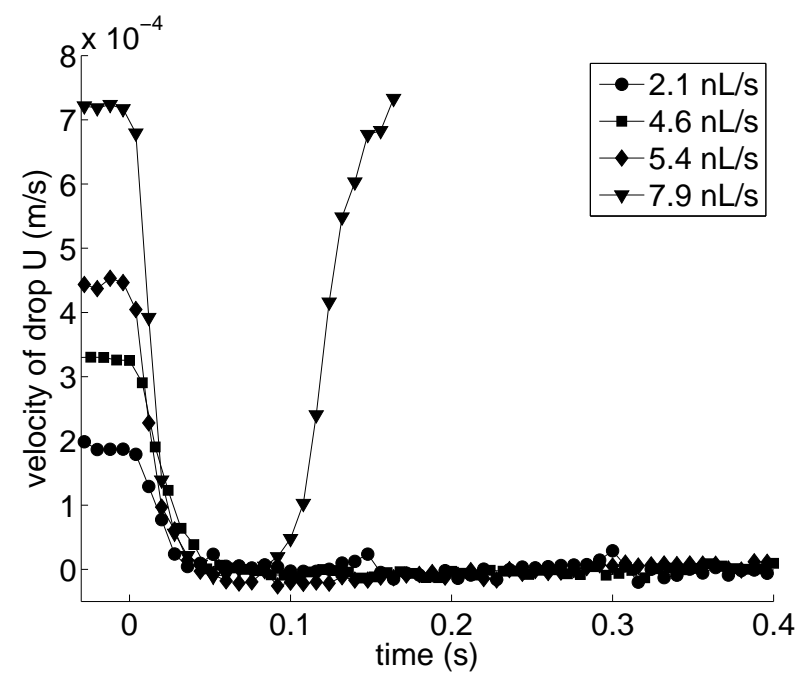

Figure 6: Time evolution of the drop velocities $U$ as they reach the laser at four different flow rates $2.1<Q<7.9 \mathrm{~nL} / \mathrm{s}$. The laser power is $P=153 \mathrm{~mW}$.

We focus our attention on the setup time for the flow field inside the drop: A characteristic velocity near the tip is obtained by equating the flux $\varphi(t)$ that flows between the roll centers away from the hot region with the flux through the lines $d$ that joins the centers of the rolls and the interface. In this way, the accuracy of the velocity data away from the hot region is used to obtain the time evolution of the velocity near the tip, since no flux crosses the interface itself. In practice, $\varphi(t)$ is calculated along the line $\ell$ in Figure $5 \mathrm{~b}$, as the sum of the normal velocities in the reference frame of the drop. The velocity near the tip $v$ is estimated by equating $\varphi=2 v d$, which yields $v(t)=\varphi(t) /(2 d)$.

As the drop reaches the laser position, $v(t)$ displays a rapid increase before reaching a saturation value, as shown in Figure 7a. Here, the time $t=0$ is the same as for the drop velocity data $U(t)$ in Figure 6, indicating that the onset of the roll motion occurs simultaneously with the drop deceleration. This data were fit with an exponential function, $v_{f i t}=v_{\infty}\left(1-e^{-t / \tau_{v}}\right)$, yielding two important physical parameters: the saturation velocity $v_{\infty}=1.3 \pm 0.2 \mathrm{~mm} . \mathrm{s}^{-1}$ which does not display any dependence on $P$ or $Q$ within the accuracy of our measurements, and the character- 
istic time $\tau_{v}$ which is also independent of $P$ but decreases with $Q$ from 20 to $6 \mathrm{~ms}$, as shown in Figure $7 \mathrm{~b}$. This time correlates very well with the time required for the drop to travel a distance equal to the laser waist, $\tau_{U}=\omega_{0} / U_{0}$.

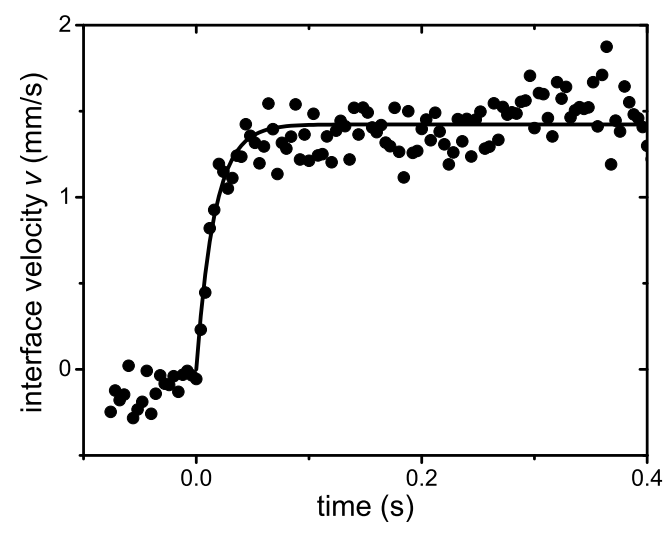

(a)

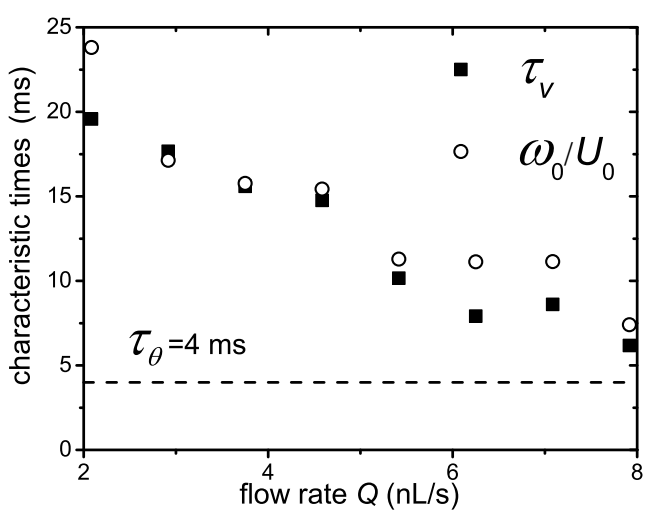

(b)

Figure 7: (a) Time-evolution of the roll velocity $v(t)$, in the drop reference frame, as the drop reaches the laser position. Solid line shows an exponential fit to the data. $P=153 \mathrm{~mW}$ and $Q=2.1 \mathrm{~nL} / \mathrm{s}$. (b) Full squares: Characteristic time $\tau_{v}$, obtained from the exponential fits of $v(t)$, as a function of flow rate $Q$. Hollow circles: time to cross the laser spot, $\omega_{0} / U_{0}$. Dotted line: typical heating time $\tau_{\Theta}$.

Several links can be drawn between the above measurements in order to shed light on the underlying physics, as discussed in the next section.

\section{Discussion}

\section{Two conditions for blocking a drop}

The first conclusion that can be reached from the above measurements is a confirmation of the hydrodynamic origin of the force that is applied on the drop. Indeed, the simultaneity of the onset of the rolls with the drop deceleration is a good indication that the two phenomena are linked.

These results indicate two independent mechanisms that determine the blocking of the drops: 
The first is associated with rapidly advancing drops which could not be blocked regardless of the laser power. In this case, the interface passes the laser spot in a time shorter than the time $\tau_{\theta}$ necessary to produce the thermal gradient, indicated by the dashed line in Figure $7 \mathrm{~b}$. The heating is therefore too slow to fully generate the recirculation rolls and block the drop, which slows down at the laser before accelerating back to its initial velocity.

The second limitation applies for slowly moving drops and is set by the magnitude of the force that can be generated for a given laser power, as indicated by the increasing $P^{\min }(Q)$. This interpretation is further confirmed by the results of Figure $4 \mathrm{~b}$. Indeed, the time to cross the laser $\tau_{U}$ when it is defocused is increased but the temperature gradient is smaller than in the focused case. $^{22}$ The increase of $P^{\mathrm{min}}$ with beam size therefore indicates that the limiting factor originates from the force magnitude rather than the setup time, as long as $Q<Q^{\max }$.

\section{Role of the confinement}

It has been shown that the velocity field associated with the capillary driven stress consists of two counter-rotating rolls in the outer fluid, ${ }^{12}$ in complement to the two inner rolls visualized in Figure 5a. In a confined geometry such as ours, the resulting stress field can be decomposed into two contributions: one associated with the recirculations near the tip of the drop and the other due to the strong shear in the thin oil films between the moving interface and the lateral walls (see Figure 5c).

Near the laser position, the force can be estimated by extracting the interface velocity $v=$ $1.3 \mathrm{~mm} / \mathrm{s}$ from the PIV measurements and using the model of an unconfined circular drop. ${ }^{12}$ The resulting force is $F_{t i p} \simeq 2 \eta_{o} v r \sin ^{2} \theta=200 \mathrm{pN}$, where $r=w / 2=50 \mu \mathrm{m}$ is the radius of curvature and $\theta=\pi / 4$ is the angular position of the roll centers, taken from Figure 5a. This force is far too small to account for the experimentally measured value of $180 \mathrm{nN}$.

In the present geometry where the drop almost fills the channel width, the viscous shear stress in the lateral films should also be taken into account. Indeed, since the interface is driven by the 
surface tension gradient towards the laser position, it exerts a reciprocal stress on the lateral fluid films away from the laser (see Figure 5).

The magnitude of this force may be estimated as the product of the viscous shear stress in the oil film times the area of the film, yielding: $F_{f i l m s}=\eta_{o} v_{s} / e .2 h L$. Here, $v_{s}=0.15 \mathrm{~mm} / \mathrm{s}$ is the mean value of the tangential velocity along the length of the drop, measured from the PIV, $h$ the channel depth, $L$ the length of the drop, and $e$ is the unknown film thickness. This film thickness can be estimated from the asymptotic analysis of Wong et al., ${ }^{23}$ for our aspect ratio $(w / h=2)$, as $e / h=0.45(3 C a)^{2 / 3}$, where the capillary number is $C a=\eta_{o} v_{s} / \gamma=9 \cdot 10^{-5}$ and yields a thickness $e=0.1 \mu \mathrm{m}$. The force can now be calculated for a $330 \mu \mathrm{m}$-long drop and we obtain $F_{\text {films }}=160 \mathrm{nN}$, in remarkable agreement with the measured force. This contribution to the total force is orders of magnitude larger than the value for $F_{t i p}$ which can be neglected. Moreover, this analysis also accounts for the effect of the drop length: it explains why longer drops were easier to block than shorter ones.

\section{Marangoni stresses and interface velocity}

The velocity of the interface $v$ measured above can be related to the surfactant and temperature distributions by considering a portion of interface, near the tip of the drop, of local radius of curvature $w / 2$. The Marangoni condition can be written from the continuity of the shear stresses at the interface, providing a relation between the normal velocity gradients and the tangential surface tension gradient:

$$
\left.\eta_{o} \frac{\partial v_{\theta}^{o}}{\partial r}\right|_{\text {interface }}-\left.\eta_{w} \frac{\partial v_{\theta}^{w}}{\partial r}\right|_{\text {interface }}=\frac{2}{w} \frac{\partial \gamma}{\partial \theta}
$$

where $\eta_{w}$ and $\eta_{o}$ are the water and oil viscosities, $v_{\theta}{ }^{w}$ and $v_{\theta}^{o}$ are the tangential velocities in the water and oil, respectively, and $r$ and $\theta$ the radial and azimuthal directions.

The typical length scale for the radial variation of the velocity in both phases is the distance 
$d$ between the interface and the roll centers (see Figure 5). A dimensional analysis can therefore be used to write the interface velocity $v$ as

$$
\left(\eta_{o}+\eta_{w}\right) \frac{v}{d} \sim \frac{2}{w} \frac{\Delta \gamma}{\Delta \theta}
$$

where the $\sim$ symbol indicates order of magnitude scaling and the $\Delta$ indicates a variation in a given variable. As detailed earlier, the change of the surface tension is due to both temperature and surfactant concentration variations. One can write as a first approximation

$$
\left(\eta_{w}+\eta_{o}\right) \frac{v}{d} \sim \frac{2}{w}\left(\gamma_{T} \frac{\Delta T}{\Delta \theta}+\gamma_{\Gamma} \frac{\Delta \Gamma}{\Delta \theta}\right)
$$

where the thermal and solutal contributions can be distinguished.

In the vicinity of the laser, the two mechanisms act in opposition since the increase in temperature decreases the surface tension $\left(\gamma_{T} \Delta T / \Delta \theta<0\right)$, while a depletion of surfactants, as shown in Figure 3, increases the surface tension $\left(\gamma_{\Gamma} \Delta \Gamma / \Delta \theta>0\right)$. Our visualization of the direction of the interface velocity indicates that the surface tension increases near the laser, implying that the solutal Marangoni effect dominates over the purely thermocapillary effect. However, a purely thermal Marangoni effect would produce a velocity $v_{t h}=\frac{2 d \gamma_{T}}{w\left(\eta_{w}+\eta_{o}\right)} \Delta T / \Delta \theta=93 \mathrm{~mm} / \mathrm{s}$ for a temperatue variation $\Delta T / \Delta \theta=20^{\circ} \mathrm{C} / \mathrm{rad}$. When compared with the measurements of $v_{\infty}=1.3 \mathrm{~mm} / \mathrm{s}$, this suggests that the two antagonistic effects almost compensate, with a slight imbalance in favor of the solutal contribution. This is consistent with other examples of thermo-solutal competition where the thermal and solutal Marangoni mechanisms are almost in exact balance. ${ }^{10,11}$

\section{Summary}

The pushing of microfluidic drops by laser heating is a surprising phenomenon that can potentially play a major role in the lab-on-a-chip technologies, if the technique can be applied in 
practical situations. This study provides the first detailed measurements concerning the physical processes at play, namely the physical chemistry aspects related to the force generation and the force magnitude.

Two practical limitations are found for achieving total blocking of moving drops. The first concerns slowly moving drops for which the heating is sufficiently fast to produce the thermocapillary flow. In this case the blocking is limited by the magnitude of the force which increases with laser power, reaching $300 \mathrm{nN}$ for our geometry and drop size. The second limitation is due to the time necessary to heat the interface, which prevents rapidly moving drops from being completely stopped. The temporal criterion that must be kept in mind is that the interface should not cross the laser waist faster than the time required to reach necessary temperature gradient, a few ms in our experiments.

The practical values of these two limits can be pushed by intelligent design of the microchannel geometry. Indeed, drops that are longer are easier to stop, suggesting that squeezing a drop by reducing the channel width can be used to increase the blocking force. On the other hand, widening the microchannels leads to a low linear velocity for a constant flow rate. These two apparently contradictory requirements can either be applied separately, or they can be combined by placing discrete pillars for guiding droplets in a wide channel, as shown recently. ${ }^{27}$ Furthermore, the value of the heating time can be manipulated by varying the volume of fluid that is heated, for example by reducing the channel thickness.

An important question remains, however, concerning the precise mechanism responsible for the micelle migration and their subsequent local depletion near the hot spot. Several hypotheses can be emitted, for example through the effect of optical forces which can act on the surfactant ${ }^{28}$ or through thermophoresis which would radially displace them away from the hot spot. ${ }^{29-31}$ An observation in favor of this last hypothesis is the ring-shaped accumulation built up during the trapping period that partially remains adsorbed on the walls, a similar feature to what is obtained in Braun and Libchaber, ${ }^{32}$ which was attributed to a thermophoretic effect coupled with 
a thermal convection. However, the details of the mechanism are probably best dealt with through numerical simulations of the coupled transport equations, which can explore the unsteady flows, temperature and concentration fields in detail.

\section{Experimental}

\section{Microchannels and microfluidics}

The fabrication of the microchannels is performed using standard soft lithography techniques. ${ }^{33}$ Briefly, a mold of the channel is microfabricated in photoresist (Laminar E7800, Eternal Technology Corp.) by conventional photolithography. A replica of this master is then molded with a $5 \mathrm{~mm}$ layer of liquid polydimethylsiloxane (PDMS, Sylgard 184, Dow Corning), which is then cured at $65^{\circ} \mathrm{C}$ for $4 \mathrm{~h}$. After curing, the PDMS replica is peeled off and holes are punched at the channel inputs and outlets. The patterned PDMS block is then sealed against a bare glass slide, after an oxidizing plasma treatment that promotes strong adhesion between the parts.

The microchannel geometry is shown in Figure 1. It has a total length of $15 \mathrm{~mm}$, a width $w=100 \mu \mathrm{m}$ and a thickness $h=50 \mu \mathrm{m}$. It has three inputs which are connected to three syringe pumps that provide independent control of the different flow rates. The main channel and the second side-channel allow the flow of an organic solution at flow rates $Q_{\mathrm{oil}}^{(1)}$ and $Q_{\mathrm{oil}}^{(2)}$, respectively, while an aqueous suspension flows through the first side-channel (flow rate $Q_{\text {water }}$ ). Drops of water in oil are formed at the first T-junction with a size $L$ set by the two flow rates $Q_{\mathrm{oil}}^{(1)}$ and $Q_{\text {water. }}$ Adjusting $Q_{\text {oil }}^{(2)}$ then allows us to vary the total flow rate $Q=Q_{\text {water }}+Q_{\text {oil }}^{(1)}+Q_{\text {oil }}^{(2)}$ and the distance between the drops without affecting the drop formation. In the experiments described below $Q_{\text {oil }}^{(1)}$ and $Q_{\text {water }}$ are kept constant at $0.830 \mathrm{~nL} / \mathrm{s}$ and $0.417 \mathrm{~nL} / \mathrm{s}$ respectively, so that the drop lengths considered are in the range $L=330 \pm 20 \mu \mathrm{m}$.

The laser blocks the drops in a test section, shown on the downstream side of the figure, which is connected in parallel to a bypass through three $20 \mu \mathrm{m}$-wide channels. These structures 
act as filters, allowing the oil to flow through them while forcing the drops to continue in the test channel ${ }^{26}$. The hydrodynamic resistance of the bypass is measured by flowing an aqueous suspension of tracer particles into the device and measuring the relative flow rates in the bypass and the test section, keeping in mind that the pressure gradient acting on the test section is the same as for the bypass. Since the resistance of the test section can be calculated with precision from the Poiseuille law for a rectangular channel ${ }^{34}$ (cross-section $h \times w$ and length $L_{t}=750 \mu \mathrm{m}$ ), the ratio of flow rates yields the resistance of the by-pass to be $R^{b}=7.210^{12} \mathrm{~Pa} \mathrm{~s} \mathrm{~m}^{-3}$.

The organic solution consists of hexadecane (Sigma-Aldrich, viscosity $\eta_{o}=3 \mathrm{mPa} . \mathrm{s}$ ) as the solvent and an oil-soluble surfactant (Sorbitan monooleate or Span80, Sigma-Aldrich) at $2 \mathrm{wt}$ $\%$. The surfactant Span80 has a neutral polar head and an unsaturated linear tail of 17 carbons, making it quasi-insoluble in water, with a water/oil partition coefficient of $5.510^{-4} .{ }^{35}$ The aqueous solution is ultra pure water (viscosity $\eta_{w}=1 \mathrm{mPa} . \mathrm{s}$ ) which contains a suspension of green-fluorescent latex beads of diameter $0.5 \mu \mathrm{m}$ (Molecular Probes, $2 \%$ solids) at a concentration of $5 \mu \mathrm{L} / \mathrm{mL}$. The beads are used as flow tracers, allowing the velocity field inside the drop to be measured using a commercial Particle Image Velocimetry (PIV) software (Davis, LaVision).

\section{Optics}

The experiments are conducted using an inverted microscope (Nikon TE2000) equiped with an epi-fluorescence setup. Image sequences of the drop interface or of the fluorescent beads are acquired through a 10x objective (Nikon, NA=0.3) with a fast camera (Photron Fastcam 1024PCI) at a frame rate of 250 frames per second. In this way, the velocity field within the drop $\mathbf{u}(x, y, t)$ is computed by cross-correlation between two successive images separated by a $4 \mathrm{~ms}$ delay, with a spatial resolution of $40 \times 100$ vectors. This corresponds to a vertically averaged velocity field since the objective's depth of field is larger than the channel depth.

The laser used in this study is an infrared (IR) fiber-coupled diode laser (Fitel Furukawa FOL1425) emitting a Gaussian beam with wavelength $\lambda=1480 \mathrm{~nm}$. The fiber is connected to 
a colimator (Oz-optics) which yields a parallel beam, $2.2 \mathrm{~mm}$ in waist. After going through a dichroic mirror, the beam is focused through the microscope lens onto the sample and care is taken to insure that the visible light is focused on the same image plane as for the IR beam. After the objective and a glass slide, the beam waist is calculated to be $\omega_{0}=5.3 \mu \mathrm{m}$ and the total power $P$ that reaches the sample spans the range 55 to $165 \mathrm{~mW}$. Finally, near-infrared absorption measurements show that the energy of the laser beam is absorbed mainly by the water. Oil absorbs a hundred times less than water, PDMS a thousand times less, and glass even less than PDMS. The absorption length in water is measured to be $k^{-1}=400 \mu \mathrm{m}$, in good agreement with literature. ${ }^{36}$

When specified, the beam width $\omega$ was varied by defocusing the objective by different distances $z$ along the optical axis. $\omega$ is calculated as $\omega^{2}=\omega_{0}^{2}\left[1+\left(n z / z_{0}\right)^{2}\right]$ with $z_{0}=\pi \omega_{0}^{2} / \lambda$ and $n=1.5$ the glass refractive index. Note that $z$ can be either positive or negative with reference to the focused position, depending on whether the microscope objective was raised or lowered.

\section{Temperature field}

In a parallel study, the spatio-temporal evolution of the temperature field in a thin water layer heated by the laser beam was measured and compared with simulations. We summarize the results here and refer the reader to the acompanying article for the complete details. ${ }^{22}$ The profile of the temperature rise in a water layer confined between two glass slides takes a Lorentzian shape $T(r, t)=\Theta(t) /\left[1+(r / \sigma(t))^{2}\right]$, where $\Theta(t)$ is the temperature at the laser position and $\sigma(t)$ the half-width at half-height of the Lorentzian, while $r$ and $t$ represent the radial distance and time, respectively. Two important time scales, both independent of the laser power, were observed: a fast time scale $\tau_{\Theta}=4 \mathrm{~ms}$, required to reach the steady value of $\Theta$ and the maximum thermal gradient, and a slow time scale $\tau \approx 30 \mathrm{~ms}$ which is associated with the setup of the steady state temperature profile. With a laser power $P=100 \mathrm{~mW}$ and in a $28 \mu \mathrm{m}$ deep water layer, the steady state values of $\Theta$ and $\sigma$ are $60^{\circ} \mathrm{C}$ and $20 \mu \mathrm{m}$ respectively. Numerical simulations show 
the temperature increase is lower, for equal power, if the laser is focused to a larger waist $\omega$, and that replacing one of the glass slides with a PDMS wall has little influence on the thermal field.

In the present experiments where the laser is only absorbed in the water drops, the maximum temperature is affected in two ways: On one hand, the larger sample depth increases the absorbed power thus leading to increased temperature rise. On the other hand, the laser only partially overlaps the drop and is not absorbed by the hexadecane, therefore tending to decrease the maximum temperature in a non trivial way. However, even though the temperature at the laser position cannot be accurately estimated, it is expected to increase with the laser power and decrease when the laser is defocused. Of the remaining parameters, we note that $\tau_{\Theta}$ will vary with the depth of the heated layer and is expected to be larger in the present channels than in the thinner layer studied by Cordero et al. . ${ }^{22}$

Finally, the flows are weak enough to neglect heat transfer by convection. Indeed, the thermal Peclet number $\mathrm{Pe}=U L / D_{T}$ can be estimated using the following values: $U=1 \mathrm{~mm} / \mathrm{s}$ is the velocity scale of the fluid, $L=\sigma \simeq 20 \mu \mathrm{m}$ is the length scale of the hot region and $D_{T}$ is the thermal diffusion coefficient $\left(D_{T}=1.310^{-7}\right.$ and $8.810^{-8} \mathrm{~m}^{2} / \mathrm{s}$ for water and hexadecane respectively). These values yield $P e=0.2$, implying that the heat transfer is dominated by diffusion.

\section{Acknowledgement}

The authors acknowledge useful discussions with Antigoni Alexandrou and Axel Buguin, and the help of the LOB at Ecole Polytechnique for the infra-red absorption measurements. The project benefited from funding by the convention $X-D G A$. EV was funded by a CNRS postdoctoral fellowship. MLC was funded by an EADS Corporate Foundation fellowship and by MIDEPLAN. 


\section{References}

(1) Young, N.; Goldstein, J.; Block, M. J Fluid Mech (1959), 6, 350-356.

(2) Brzoska, J.-B.; Brochard-Wyart, F.; Rondelez, F. Langmuir 1993, 9, 2220-2224.

(3) Sammarco, T.; Burns, M. AIChE J 1999, 45, 350-366.

(4) Lajeunesse, E.; Homsy, G. Phys. Fluids 2003, 15, 308-314.

(5) Garnier, N.; Grigoriev, R.; Schatz, M. Phys. Rev. Lett. 2003, 91, 054501.

(6) Bush, J. J. Fluid Mech. 1997, 352, 283-303.

(7) Sumino, Y.; Magome, N.; Hamada, T.; Yoshikawa, K. Phys. Rev. Lett. 2005, 94, 068301.

(8) Darhuber, A.; Troian, S. Annu. Rev. Fluid Mech. 2005, 37, 425-455.

(9) Mazouchi, A.; Homsy, G. Phys. Fluids 2000, 12, 542-549.

(10) Chen, J.; Stebe, K. J. Fluid Mech. 1997, 340, 35-59.

(11) Gugliotti, M.; Baptista, M. S.; Politi, M. J. Langmuir 2002, 18, 9792-9798.

(12) Baroud, C. N.; Delville, J.-P.; Gallaire, F.; Wunenburger, R. Phys. Rev. E. 2007, 75, 046302.

(13) Baroud, C. N.; Robert de Saint Vincent, M.; Delville, J.-P. Lab Chip 2007, 7, 1029-1033.

(14) Robert de Saint Vincent, M.; Wunenburger, R.; Delville, J.-P. Applied Physics Letters 2008, $92,154105$.

(15) Campanelli, J. R.; Wang, X. J Coll Int Sci 1999, 213, 340-351.

(16) Adamson, A.; Gast, A. Physical Chemistry of surfaces, 6th ed.; Wiley-Interscience: New York, NY, 1997. 
(17) Espeau, P.; Ceolin, R. Thermochimica Acta 2006, 445, 32-35.

(18) Levich, V. Physicochemical Hydrodynamics; Prentice-Hall: Englewood cliffs, NJ, 1962.

(19) Eastoe, J.; Dalton, J. Advances in colloid and interface science 2000, 85, 103-144.

(20) Johner, A.; Joanny, J. Macromolecules 1990, 23, 5299-5311.

(21) Karstens, T.; Kobs, K. Journal of Physical Chemistry 1980, 84, 1871-1872.

(22) Cordero, M.; Verneuil, E.; Gallaire, F.; Baroud, C. Submitted to Phys. Rev. E 2008.

(23) Wong, H.; Radke, C.; Morris, S. J. Fluid Mech. 1995, 292, 71-94.

(24) Fuerstman, M.; Lai, A.; Thurlow, M.; Shevkoplyas, S.; Stone, H.; Whitesides, G. Lab on a Chip 2007, 7, 1479-1489.

(25) Jousse, F.; Lian, G.; Janes, R.; Melrose, J. Lab Chip 2005, 5, 646-656.

(26) Cristobal, G.; Benoit, J.-P.; Joanicot, M.; Ajdari, A. Appl Phys Lett 2006, 89, 034104.

(27) Niu, X.; Gulati, S.; Edel, J.; deMello, A. Lab Chip 2008, 8, 1837-1841.

(28) Bar-Ziv, R.; Moses, E. Physical Review Letters 1994, 73, 1392-1395.

(29) Giglio, M.; Vendramini, A. Physical Review Letters 1977, 38, 26-30.

(30) Rauch, J.; Kohler, W. Physical Review Letters 2002, 88, 185901.

(31) Vigolo, D.; Brambilla, G.; Piazza, R. Phys. Rev. E. 2007, 75, 040401.

(32) Braun, D.; Libchaber, A. Physical Review Letters 2002, 89, 188103.

(33) Xia, Y. N.; Whitesides, G. M. Ang. Chem. Int. Ed. Engl. 1998, 37, 551-575.

(34) Stone, H.; Stroock, A.; Ajdari, A. Ann. Rev. Fluid. Mech. 2004, 36, 381-411. 
(35) Peltonen, L.; Yliruusi, J. J Coll Int Sci 2000, 227, 1-6.

(36) Duhr, S.; Arduini, S.; Braun, D. The European Physical Journal E 2004, 15, 277-286. 